\title{
Importancia de las publicaciones académicas: algunos problemas y recomendaciones a tener en cuenta
}

\author{
The importance of academic publications: Some problems \\ and recommendations to keep in mind
}

\author{
Francisco Ganga Contreras ${ }^{1 *}$, Lorena Paredes Buzeta ${ }^{2}$, Liliana Pedraja-Rejas ${ }^{3}$
}

\section{RESUMEN}

Las publicaciones académicas en revistas científicas son la viva representación de la generación de conocimiento, proceso que constituye uno de los principales roles que debe cumplir una universidad; sin embargo, a la hora de publicar los hallazgos de las investigaciones realizadas, se pueden presentar determinados problemas. En este orden de cosas, el objetivo central de este trabajo es efectuar ciertas reflexiones en relación con la relevancia que tienen las publicaciones académicas en la sociedad del conocimiento, y adicionalmente presentar algunas dificultades que se evidencian en el correspondiente proceso, complementadas con sugerencias para afrontar estos obstáculos.

Desde el punto de vista de los contenidos se comienza por presentar aspectos contextuales que posibilitan plantear la problemática, luego se pasa revista a los problemas más habituales que enfrentan los autores, a la hora de presentar un artículo para una posible publicación; entre estos se citan: tiempo de demora de la publicación, abuso de las autocitas, opacidad en la aceptación de artículos, incompetencias en la revisión de los artículos y faltas de rigor en el cálculo del factor de impacto. Finalmente, sobre la base de los problemas identificados, se entregan algunas recomendaciones para que los autores tengan en consideración a la hora de publicar en revistas científicas.

Palabras clave: Autocitación, publicaciones académicas, factor de impacto, revistas científicas.

\begin{abstract}
Academic publications in scientific journals are the embodiment of knowledge generation, a process that is one of the major roles that a university must achieve. However, when publishing the findings of conducted researches, certain problems can emerge. The present paper begins by setting contextual aspects that allow to present the topic, then reviews the most common problems faced by authors when submitting an article for possible publication; among these they are: delay time of publication, abuse of self-citations, opacity in accepting articles, incompetence in reviewing articles and lack of rigor in the calculation of the impact factor. Finally, based on the problems identified previously, some recommendations are made, to take into consideration when publishing in scientific journals.
\end{abstract}

Key words: Self-citation, academic publications, impact factor, scientific journals.

\section{Introducción}

Una de las tareas primordiales que debe asumir la universidad, dice relación con la generación de nuevo conocimiento, el que debe estar al servicio de la sociedad de la que forma parte, debido a que son estas entidades las que deben asumir la responsabilidad de generar e irradiar la vida intelectual de su comunidad académica. En la actualidad nadie discute que el conocimiento se ha transformado en un factor determinante en el éxito de las sociedades, pues es el basamento de la construcción social y de su correspondiente vertebración con todo el tejido social. Hoy se está muy lejos de aquellos tiempos remotos donde el conocimiento se transmitía de generación en generación por medio del habla, y por lo tanto este quedaba circunscrito a la memoria de las familias, clanes, tribus o grupos de familias; o a posteriori, en los intramuros de las entidades del saber, las escuelas o las universidades; esto

\footnotetext{
Departamento de Ciencias del Desarrollo, Universidad de Los Lagos-Campus Santiago. Santiago, Chile.

Gotschlich \& Gotschlich Consultores S.A. Santiago, Chile.

3 Centro de Estudios CEUTA, Universidad de Tarapacá, Arica.

* Autor para correspondencia: fganga@ulagos.cl
}

Fecha de Recepción: 10 Julio, 2015.

Fecha de Aceptación: 29 Agosto, 2015. 
implicaba que los intercambios de información en esa época eran extremadamente engorrosos, y en muchos casos se mantenían en núcleos difíciles de penetrar. Con el paso del tiempo, esta realidad se fue modificando sustantiva y especialmente a partir del "hombre Gutemberg" -como lo expresa Riaza (2002)- comienza a hacerse más asequible y a desplazarse más velozmente.

Uno de los dispositivos que han facilitado el acceso a nuevos saberes son las revistas académicas o científicas, las que se han transformado en una de las herramientas o vehículos más efectivos, sofisticados y rigurosos para dar a conocer el conocimiento que se está gestando en todas las áreas disciplinarias, y esto, obviamente no es una excepción para el ámbito de la administración organizacional.

Considerando esta realidad, este trabajo tiene como finalidad cardinal reflexionar respecto de la importancia que tienen las publicaciones académicas como diseminadoras del conocimiento y también considerar las dificultades que pueden presentarse en el respectivo proceso; junto con lo anterior, se pretende asimismo, proporcionar recomendaciones que coadyuven la elección de la revista más apropiada para que los investigadores puedan publicar los resultados de sus indagaciones. Este propósito se pretende lograr, utilizando primordialmente fuentes secundarias, lo que implica la revisión de artículos e información aparecida en internet.

\section{Antecedentes contextuales}

En 2006, Jaramillo expresaba que las universidades deben salir de sus claustros y ponerse al servicio de la sociedad, al mismo tiempo agregaba que la ciencia debe salir de los laboratorios y ser enseñada y democratizada para que la sociedad toda le dé su lugar, su legitimidad. Estas aseveraciones dan pie para argumentar que las universidades tienen dentro de sus imperativos la germinación de nuevo conocimiento, el que debe hacerse disponible a la sociedad, con instrumentos efectivos, como son las revistas científicas, donde los académicos pueden publicar sus indagaciones, como forma de artículo. En esta dirección, Jiménez (2008) argumenta que el investigador tiene la necesidad de publicar los resultados de sus hallazgos. Para ello el mundo científico cuenta con un sistema de validación constituido por investigadores que ratifica, rechaza, corrige, retroalimenta o refuta los resultados de sus investigaciones. En otras palabras, se puede adicionar que efectivamente los artículos representan resultados originales que pasan por un proceso de revisión riguroso por parte de expertos en los temas que se tratan en ellos y, en consecuencia, proporcionan una mayor confiabilidad de las ideas que ellos exponen. En promedio, un artículo científico de una revista registrada en un índice está expuesto a la revisión de al menos dos evaluadores anónimos (doble ciego) en muchos casos tres (triple ciego) - que son miembros de la comunidad científica, versados o especialistas en el tema que se pretende publicar- más las respectivas opiniones del director o editor académico y administrativo; mecanismos que habitualmente no se utilizan en la producción de un libro.

Se podría decir entonces, que estos dispositivos operan en la práctica como un verdadero sistema de aseguramiento de la calidad de la producción científica, siendo una refrendación de la producción intelectual que genera una universidad o centro de investigación, por medio de sus académicos.

Origgi y Ramello (2015) discuten acerca de cómo puede variar el rol de las publicaciones científicas, ya que si bien en un comienzo nacieron por la necesidad de compartir los saberes, hoy en día esto se puede ver a veces opacado por el fin de obtener primordialmente los incentivos económicos; todos estos elementos son importantes, pero lo más relevante debiera ser el incentivo que tienen los investigadores de resolver problemas que afectan a la comunidad, aportando decididamente al progreso de sus respectivos países y del mundo en general.

A pesar de lo expresado anteriormente, no se puede desconocer que existen miembros de las comunidades académicas que no están de acuerdo con esta forma de acceder y promover el conocimiento científico y han emitido profusas críticas a este tipo de revistas, sin embargo, no se puede desconocer que el prestigio de muchos de ellos se debe precisamente a estos instrumentales, pero también se puede argumentar que las revistas académicas que han logrado mucha reputación y ascendencia son justamente aquellas que han conseguido ser reconocidas por índices definidos por entes constituidos por investigadores que respaldan la excelencia de estas, aceptando su incorporación a determinadas bases de datos (por eso se conocen como revistas indexadas o indizadas).

\section{Problemas más comunes}

En general las dificultades que habitualmente se producen cuando se trata de publicar un artículo 
en una revista académica, pueden ser agrupados en dos grandes categorías: inconvenientes coligados a las revistas y problemas exógenos o de contexto.

\section{Problemas asociados a las revistas}

Como toda creación humana, el ámbito de las revistas no está al margen de algunas vulnerabilidades o problemáticas, que son necesarias de conocer. A continuación se hace referencia a algunas de estas debilidades.

\subsection{Tiempo de demora en la publicación}

Como la comunidad académica internacional ha entendido que es muy relevante publicar en revistas indexadas los conocimientos que se vayan generando, de tal forma que estos no queden encerrados en los laboratorios o en las bibliotecas de los académicos, investigadores o tesistas, se ha producido un incremento sustantivo en la oferta de artículos académicos, tanto es así que en muchos casos, las revistas reciben cantidades que superan en diez veces o más los artículos que necesitan para un número determinado. Esta realidad ha provocado que no existan suficientes evaluadores anónimos con el tiempo apropiado para arbitrar en un plazo razonable los trabajos que reciben. Como consecuencia de ello, los lapsos de demora-desde que se envía el artículo para evaluación, hasta su publicación-se han incrementado exponencialmente. En este sentido, en un estudio realizado por Cruz (2013), en revistas especializadas en gerencia, detectó que el tiempo de respuesta de la primera ronda de revisión puede tomar hasta 85 días (casi tres meses). Un artículo que pasa por cuatro rondas de revisión puede tomar un tiempo de 224 días (7,5 meses) antes de ser aceptado finalmente para su publicación. En la Figura 1 se puede observar gráficamente lo expresado en forma previa.

$\mathrm{Si}$ a lo anterior se le añade el hecho de que muchas de las revistas indexadas llevan por adelantado sus números en alrededor de un año, la esperanza de publicar se alarga a prácticamente veinticuatro meses.

\subsection{Abuso de las autocitas}

La autocitación se define como el número de veces que una revista se cita a sí misma en las referencias bibliográficas de sus artículos (De Vito
2006). Cuando existen redes académicas, o trabajos que tienen continuidad investigativa lógica, no tiene nada de malo referenciar y sustentar los trabajos con los avances previos que el propio autor o los colegas miembros de las redes han realizado, de hecho, realizar estas acciones resultan deseables, son legítimas y necesarias, especialmente cuando se está frente a líneas investigativas novedosas (MolinaMontenegro 2010, Ardanuy 2012). El problema se puede suscitar cuando se generan colusiones de autocitas, establecidas primordialmente por los editores de las revistas o por grupos de autores, que se colocan de acuerdo para citarse de manera indiscriminada y sin sentido investigativo entre ellos; con la única intención de afectar el factor de impacto (FI) de la revista, es decir, cuando el medio se transforma en un fin. En esta línea de análisis, según Villatoro (2013a), en el último JCR 2012, publicado el año 2013, se retiró del índice de impacto a 37 nuevas revistas, con un total acumulado de 66 revistas retiradas a esa fecha; el mismo autor agrega que estas revistas pasan por una especie de purgatorio de dos años y si logran demostrar que ya no realizan prácticas inmorales, pueden recuperar su FI.

El FI puede ser considerado como un indicador de la calidad de las revistas científicas, ya que, desde una perspectiva general, representa el número de citaciones que reciben las revistas debido a sus artículos (Schweizer, 2015). No obstante, Garfield y Pudovkin (2015) afirman que el factor de impacto de una revista no determinará el número de citaciones que tendrá en el futuro un artículo contenido en la publicación.

En todo caso, en general los estudios concuerdan en indicar que si bien existe este problema (del exceso o uso inadecuado de las autocitas), esto no debería afectar el factor de impacto.

"Thomson Reuters estudió las 5876 revistas en el JCR Science Edition 2002. Como muestra la figura que abre esta entrada, 4816 revistas $(82 \%$ del total) tenían tasas de autocitas por debajo del $20 \%$. La correlación entre el índice de impacto y la tasa de autocitas es muy baja $\left(\mathrm{R}^{2}=0,037\right)$. Un estudio de todas y cada una de las 170 categorías en el JCR Science Edition 2002 mostró que hay correlación débil entre el número de revistas en una categoría concreta y la tasa de autocitas $\left(\mathrm{R}^{2}=0,1\right)$; tampoco se observó correlación entre el puesto de una revista dentro de una categoría concreta y su tasa de autocitas" (McVeigh, 2002). 
Tabla I. Recomendaciones a considerar al momento de elegir una revista.

\begin{tabular}{lll}
\hline $\mathrm{N}^{\circ}$ & \multicolumn{1}{c}{ Criterio } & \multicolumn{1}{c}{ Explicación } \\
\hline 1 & Indexación & $\begin{array}{l}\text { Analizar si la revista está o no indexada y en qué índice (ISI, Scopus, Scielo, Latindex Catálogo, } \\
\text { y otros). }\end{array}$ \\
2 & $\begin{array}{l}\text { En idioma inglés existen mayor cantidad de revistas indexadas. Sin embargo, los investigadores } \\
\text { latinoamericanos encuentran menos obstáculos al publicar en su idioma nativo, aunque deben }\end{array}$
\end{tabular}
considerar que las revistas especializadas de países hispanohablantes son menos numerosas.

3 Cantidad de números Si la revista publica una mayor cantidad de números al año, podrían existir mayores posibilidades de acortar los tiempos de publicación.

4 Cantidad de artículos Si la revista publica una mayor cantidad de artículos por número, podrían existir mayores posibilidades de acortar los tiempos de publicación

5 Factor de impacto (FI) Es la frecuencia con la que una revista ha sido citada en un año en particular. Este indicador permite comparar revistas y evaluar la importancia relativa dentro de un mismo campo científico. Cuanto mayor sea el FI, más exigente serán las evaluaciones y más difícil la publicación.

6 Línea editorial

7 Tipo de arbitraje

8 Especificidad o especialidad de la revista

10 Lugar de la revista

11 Tipos de artículos

12 Respeto a las normas de las revistas

13 Trabajo en equipo

14 Experiencia de los autores
Es importante considerar los temas o campo disciplinar que prioriza la revista.

Usualmente la cantidad de revisores o "evaluadores ciegos" aumenta la dificultad.

Si el artículo redactado disciplinariamente es de alta calidad, quizás convenga intentar publicarlo en una revista especializada. De todos modos, si bien las revistas con una temática más amplia pueden no ser tan exigentes, reciben gran cantidad de trabajos, lo que disminuye las posibilidades de publicación.

Es muy importante considerar si la revista se publica a nivel nacional, o si es de otro país, por el tema de la endogamia académica.

Existen revistas que publican solo artículos de investigación; sin embargo, hay muchas que además publican artículos de revisión, notas técnicas, reseñas, etcétera.

Las pautas a los autores o criterios formales son predeterminadas por cada revista y su relevancia no puede ser subestimada; por lo que se deben respetar al pie de la letra.

La investigación no es para "llaneros solitarios", por lo que se debe fomentar el trabajo en equipo y el fomento de la conformación de redes de académicos interesados en generar nuevo conocimiento.

Siempre es vital considerar en los equipos investigativos, investigadores "seniors", es decir, académicos con experiencia comprobada y reconocida, que tengan a su haber proyectos de investigación adjudicados y ejecutados y varias publicaciones a "su haber". Son ellos quienes deben liderar los equipos y ser los "puntas de lanza" en los respectivos artículos.

Fuente: Diseño propio, a partir de la experiencia de los autores.

A conclusiones un tanto similares llegaron los autores Campanario y Candelario (2010), cuando sostienen que el número absoluto de autocitas que contribuyen al factor de impacto es muy bajo en algunas de estas revistas durante el período estudiado. Por tanto, no puede afirmarse más allá de toda duda que los aumentos en la variable FI en la muestra de revistas analizadas se deban a políticas deliberadas para aumentar la CFI (citas que contribuyen al factor de impacto) mediante el abuso de las autocitas. Por otra parte, en la muestra de revistas cuyo FI creció durante seis años seguidos durante los diez 


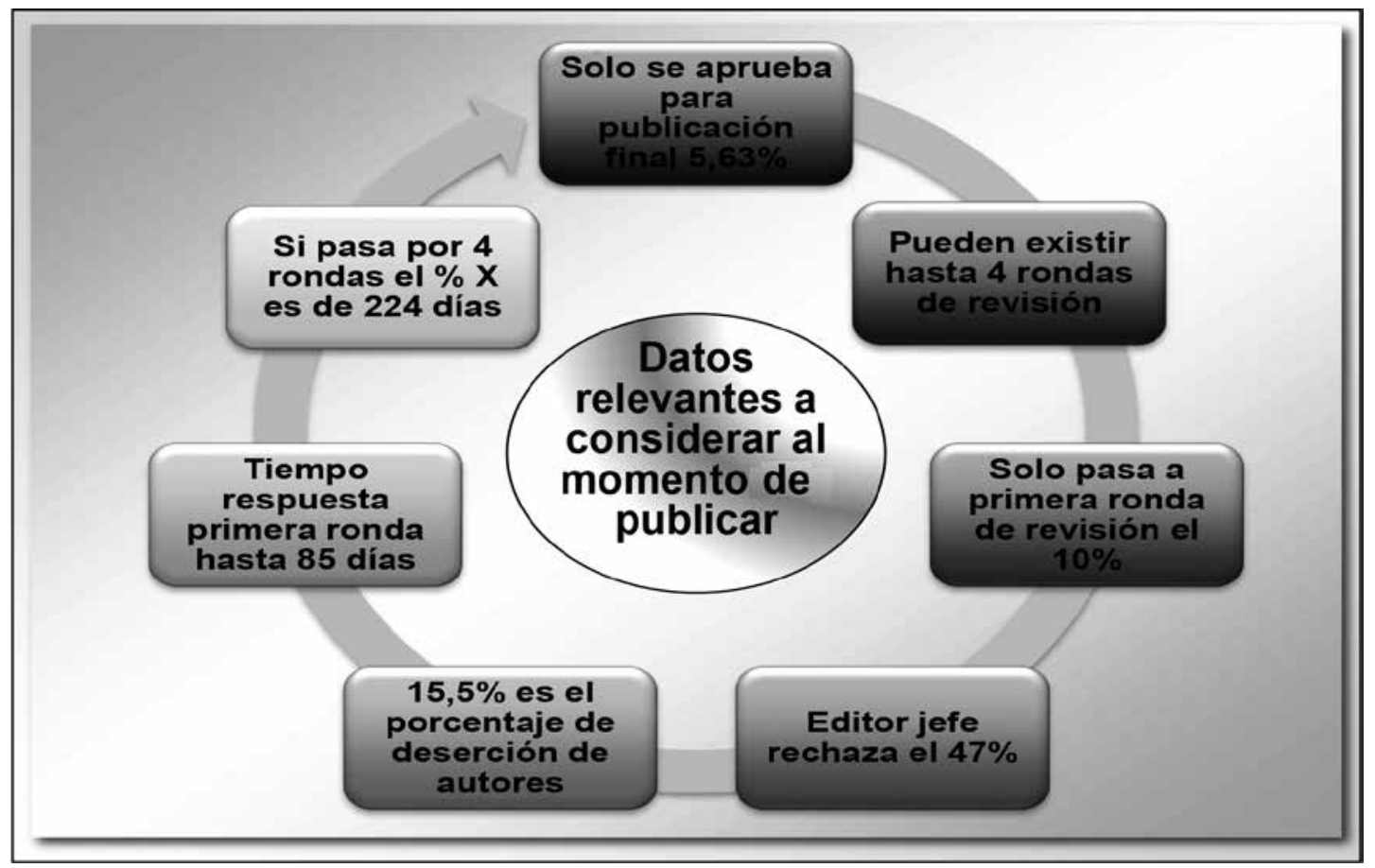

Figura 1. Tiempos a considerar en una publicación

Fuente: Diseño propio, basado en Cruz (2013)

años estudiados, el análisis de la variable ACFI no revela una influencia generalizada en el incremento de dicho indicador bibliométrico.

\subsection{Opacidad en la aceptación de artículos}

Muchas veces los académicos que se inician en las publicaciones, al no tener un "investigador senior", "maestro", "catedrático" o "titular" que los respalde, buscan al interior del sistema alguna alternativa rápida para ingresar al círculo de las revistas de calidad. Como respuesta a ellos se ha creado una mala práctica en países del tercer mundo, que consiste en crear revistas (sobre todo en línea) que son admitidas en el ISI bajo la política de apoyar al tercer mundo y no solo favorecer al primer mundo. Para prevenir esta mala práctica existe la Lista de Beall (2012), donde se recomienda a los investigadores, científicos y académicos evitar hacer negocios con los editores y revistas que están en ese registro. El año 2011 la lista estaba integrada por veintitrés editores, el año 2012 por doscientos veinticinco, esto producto de las denuncias constantes que están haciendo los académicos o investigadores. Otra forma de poder alertar a los usuarios de esta práctica es, según Jelte
Wicherts (citado en Villatoro, 2012), creando un "índice de transparencia", donde se explique en la respectiva web, de forma nítida, cuestiones como: temática, normativas o políticas específicas, lectores objetivos, procedimientos de revisión por pares, tiempos de demora aproximados y también su tasa de aceptación/rechazo. En todo caso, en general se puede observar que todas las revistas indexadas consideran aspectos como: información general de la revista (misión, indexación, dependencia institucional, etcétera), línea editorial, información sobre el editor, comité o cuerpo editorial, pautas para los autores, estadísticas, métricas, artículos publicados, y otros.

\subsection{Incompetencias en la revisión de artículos}

Tal como plantean Székely et al. (2014), hoy, producto del incremento de artículos que reciben los editores y la necesidad de someterlos con cierta rapidez a evaluación ciega, estos muchas veces cometen el error de elegir revisores que no son los más pertinentes para actuar en el rol de evaluador ciego; es más, en ocasiones estos revisores no tienen la experiencia suficiente, debido a que cuentan a su haber con muy pocas publicaciones o no son 
exactamente de la especialidad del artículo que se está evaluando; como también ocurre que estos evaluadores muchas veces no le otorgan prioridad a este trabajo y no hacen de forma correcta o completa su tarea; esto implica que se deben hacer esfuerzos para identificar aquellos evaluadores altamente dedicados e implicados con las tareas investigativas y que asumen con propiedad el rótulo de guardianes o celadores de la ciencia, como muchos académicos los han denominado. Por lo anterior, existen voces que plantean que los dobles o triples ciego sean debidamente recompensados (y no ad honorem como en la mayoría de las revistas) y que se proponga la generación de un nuevo indicador que permita medir la calidad de las revisiones y las competencias de los evaluadores. En este orden de cosas, una base de datos que permita la acumulación de manera transparente de toda esta información podría resultar ser una buena medida.

\section{Problemas asociados al contexto}

Pero no todas las dificultades que se presentan tienen como principal responsable a las revistas propiamente tales, también es factible identificar al menos dos inconvenientes que dependen más bien del contexto.

\subsection{Algunas faltas de rigor en el cálculo del factor de impacto}

De acuerdo con lo señalado por Villatoro (2013b) y publicado en el Observatorio de Revistas Científicas de Ciencias Sociales, existe una falta de rigor de Thomson Reuters al calcular el índice de impacto de una revista en el JCR. Esto se debe a que las revistas científicas publican artículos de diferentes tipos, ya sean: editoriales, noticias, artículos, revisiones bibliográficas, entre otros, y al solicitar ciertos directores que algunos de ellos no sean considerados en el cálculo del índice de impacto, este se ve incrementado.

Es así como Jump (2013) explica que el director general de Thomson Reuters ha sostenido que su compañía "no tenía control sobre la calidad de las decisiones que sus clientes hacen, y admitió que la excesiva dependencia de las citas en juzgar el desempeño académico individual habían llevado a algunas "malas decisiones".

Por su parte, Rockefeller University Press detectó errores en el cálculo del factor de impacto entregado por Thomson Reuters. Con la finalidad de comprobar la exactitud de la clasificación de algunas de sus revistas por ISI, Rockefeller University Press compró el acceso a los datos de citas de sus revistas y algunos competidores, detectando discrepancias de hasta un $19 \%$ en el cálculo del indicador. Se suma a lo anterior la poca transparencia en los datos usados y el modelo que está detrás del cálculo del Factor de Impacto (Brembs, et al., 2013).

A su vez, De Vito ya sostenía el 2006 que este índice tenía una serie de limitaciones como por ejemplo: el alto impacto de una revista no concede un alto impacto a sus artículos; el uso de un espacio de tiempo corto para el cálculo del indicador (citas a los artículos publicados solo en los dos años anteriores) en el cálculo del FI puede introducir un sesgo temporal; la base de datos a partir de la cual se calculan las citas no es completamente representativa de la producción científica internacional (existe un sesgo exageradamente favorable a las revistas angloamericanas y, en consecuencia, los artículos que no se han publicado en las revistas de estos países obtienen menos citas); no todos los artículos de una revista tienen la misma calidad (seguramente no tendrá la misma calidad una investigación multicéntrica que una carta al director); la relación entre calidad de las revistas basada en el reconocimiento del valor de la publicación por la comunidad científica por medio de las citas no es uniforme; el factor de impacto de las revistas con revisiones es alto, sin relación obligada a la calidad de los artículos; entre otras críticas.

\subsection{Comparar "peras con manzanas": índice de inmediatez y país de origen de las publicaciones}

De acuerdo con lo señalado por Aleixandre $e t$ al. (2007), "el FI no debe aplicarse para comparar disciplinas diferentes, ya que cada una tiene pautas de citación distintas". Lo que se ve reflejado en que no todos los artículos científicos comienzan a generar citas con la misma rapidez; como señala De Régules (2013), en los primeros dos años de vida de un artículo en el área de física y matemáticas generan entre $1 \%$ y $3 \%$ de las citas; en cambio en biología y afines, es entre $5 \%$ y $8 \%$.

Por otro lado, Contreras et al. (2006) señalan que la composición disciplinaria de la producción científica es similar en todos los países, pero su desempeño es diferente, ya que está correlacionado con el PIB per cápita, la inversión en I\&D a nivel 
universitario y la tasa de matrícula en educación superior. Los autores explican:

"En economía y administración también se observan diferencias entre los países latinoamericanos y el resto de los países considerados. Mientras que en Latinoamérica la participación más alta la tiene Chile con $0,8 \%$, en EE.UU. la participación es 2,2\%, en Canadá e Irlanda 1,9\% y en Nueva Zelanda $1,6 \%$. No obstante, en todos los países la producción científica de economía y administración es significativamente menor que en las ciencias básicas como química, física, biología y bioquímica y que en otras disciplinas como ingeniería, ciencias de las plantas y animales y medicina clínica (Contreras et al., 2006:340)".

De acuerdo con Contreras et al. (2006), Estados Unidos tiene un factor de impacto por sobre el promedio mundial en todas las disciplinas, es decir, sus artículos tienden a citarse en mayor cantidad, le siguen Canadá, Nueva Zelanda e Irlanda, con 19,6 y 3 disciplinas sobre el promedio. En cambio en América Latina ningún país está por sobre el promedio mundial.

\section{Recomendaciones a considerar al momento de elegir una revista}

A partir de la experiencia que existe, en la publicación de artículos académicos es factible identificar al menos once sugerencias que son determinantes al momento de elegir la revista más apropiada para enviar un artículo para una posible publicación; el detalle de estas recomendaciones puede observarse en la Tabla I.

A modo de colofón se citará el planteamiento de Heredia (2010), quien destaca que una rigurosa y concienzuda elaboración de un artículo académico, que se ciña a las normas preestablecidas por la revista y al rigor científico necesario, facilita la tarea de quienes tienen la labor de evaluar el correspondiente documento.

\section{Conclusiones}

Hoy existe una diversidad de alternativas para difundir el quehacer científico: en revistas indexadas impresas, libros con comité editorial, revistas no indexadas impresas, revistas electrónicas (indexadas y no indexadas), en actas de congresos, seminarios, entre otros. Ante tal diversidad, los investigadores se encuentran en la disyuntiva de cuál de estos medios es el más apropiado o de calidad para publicar su investigación. Esto trajo consigo la creación de indicadores bibliométricos de calidad, que buscan medir la excelencia de las revistas para la toma de decisiones de las instituciones, respecto de cuál de ellas suscribirse o en cuál los académicos deben preferentemente publicar.

Junto a lo anterior se adiciona el aumento de investigadores y, por ende, de la cantidad de artículos que se generan cada año, en este sentido el trabajo de revisión de pares, en muchos casos no se realiza correctamente, verbi gratia: no hay rigurosidad en determinar si las estadísticas, figuras y el texto son claros, o si los argumentos tienen sentido lógico, si la metodología es la apropiada, si los contenidos se ajustan a las pautas respectivas, etcétera. Por lo tanto, al parecer, se hace necesario establecer lineamientos claros sobre las buenas prácticas de publicación, las normas éticas que deben primar y cómo enfrentar los conflictos de intereses.

En todo caso, se han creado diversas revistas científicas ya sean genéricas o de especialidad, lo que ha llevado a digitalizar mucha información, existiendo actualmente distintas bases de datos que almacenan las revistas y los artículos que estas contienen, todo lo cual hace más diáfano el escrutinio que pueden recibir de la comunidad de lectores.

Otro aspecto importante a destacar es el aporte que hacen las investigaciones científicas y la divulgación de estas en revistas de especialidad al desarrollo de las respectivas sociedades, y es aquí donde los países subdesarrollados o en vías de desarrollo tienen una gran tarea, ya que la gran mayoría de estos tienen una baja inversión en investigación y desarrollo, y está comprobado que la producción científica está relacionada con el PIB per cápita, la inversión en I\&D a nivel universitario y la tasa de matrícula en educación superior.

Adicionalmente es relevante reconocer que mientras los investigadores de los países desarrollados publican en conocidas revistas de amplia circulación, los de países de pocos recursos no logran publicar más allá de sus propias fronteras. Todo ello trae consigo la denominada invisibilidad del quehacer científico de los países en vías de desarrollo y ha originado grandes debates al respecto. Si bien, la solución del problema no solo depende de las directivas de las revistas científicas, sería de mucha ayuda su apoyo y contribución para que estos investigadores de los llamados países en desarrollo logren sacar a la luz su obra. 
Por otro lado, teniendo en cuenta que la inmediatez de la información debe sentar las bases de una publicación académica, ya que la velocidad de generación de conocimiento en los últimos años se ha incrementado exponencialmente, se requiere que los comités editoriales de las revistas se ajusten a esta realidad y aceleren sus procesos de revisión y publicación de los artículos presentados por los investigadores, de tal forma de no llegar al punto en que lo que se publique ya se encuentre obsoleto.

Asimismo, no se puede dejar de subrayar que el objetivo de la comunidad científica es la creación y difusión de nuevos conocimientos, y no el indicador en sí mismo; por ello, algunos indicadores en los últimos años han sido cuestionados por su baja contribución, ya que muchos de ellos presentan graves problemas de representatividad del aporte de la revista y del artículo a la divulgación del conocimiento de punta que espera la sociedad.
Como se sabe, las universidades por su propia naturaleza son organizaciones de altos niveles de producción de conocimiento científico; y por lo mismo, es incuestionablemente relevante que esta labor de investigación se conozca fuera de la institución que la origina y la mejor manera de alcanzar tal propósito es la publicación en revistas científicas indizadas. Es así, como estudiar ciertos aspectos relacionados con la forma y fondo de las revistas resulta muy necesario, debido a que estas investigaciones se transforman en especies de monitoreo de las prácticas que están llevando a cabo estos dispositivos difusores del nuevo conocimiento que generan las universidades o los respectivos programas, centros o institutos de investigación. Es innegable que mientras mejor sea el conocimiento que se publica, mejor será la docencia que se entrega a los ciudadanos y mejores probabilidades de progreso y desarrollo tendrán los respectivos países.

\section{Literatura Citada}

Aleixandre, R.; Valderrama, J.C.; González, G.

2007. El Factor de Impacto de las Revistas Científicas: Limitaciones e Indicadores Alternativos. Revista El Profesional de la Información, 16(1): 4-11.

Ardunay, J.

2012. Breve introducción a la bibliometría. Disponible en: http://diposit.ub.edu/dspace/bitstream/2445/30962/1/ breve\%20introduccion\%20bibliometria.pdf Consultado: $31 /$ agosto/ 2015

Beall, J.

2012. Beall's List of Predatory Publishers 2013. Bajado el 20 de agosto de 2014 de: http://scholarlyoa.com/2012/12/06/ bealls-list-of-predatory-publishers-2013/

Brembs, B.; Button, K.; Munafò, M.

2013. Deep Impact: Unintended Consequences of Journal Rank. Frontiers in Human Neuroscience, 7(291): 1-33.

Campanario, J. M.; Candelario, A.

2010. La influencia de las autocitas en el aumento del factor de impacto en revistas de Ciencias Sociales. Revista española de documentación científica, 33(2): 185-200.

Contreras, C.; Edwards, G.; Mizala, A.

2006. La Productividad Científica de Economía y Administración en Chile. Un Análisis Comparativo. Revista Cuadernos de Economía, 43: 331-354.

Cruz, A. M.

2013. Cómo publicar artículos de gestión en salud en revistas de gerencia de alto índice impacto. Ciencias de la salud, 11(3): 349-365.

De Régules, $\mathrm{S}$

2013. Factor de Impacto. Disponible en: http://imagenenlaciencia. blogspot.com/2013/07/factor-de-impacto.html consultado: 23/ agosto/ 2014

De Vito, E. L.

2006. Algunas consideraciones en torno al uso del Factor de Impacto y de la Bibliometría como herramienta de evaluación científica. Revista Argentina de Medicina Respiratoria, 1 : $37-45$.

Garfield, E.; Pudovkin, A.

2015. Journal Impact Factor Strongly Correlates with the Citedness of the Median Journal Paper. Collnet Journal of Scientometrics and Information Management, 9(1): 5-14. Heredia, A.

2010. ¿Por qué y cómo publicar artículos científicos en revistas internacionales? Seminario para Autores. Elsevier Latinoamérica.

Jaramillo, A.

2006. La Universidad Frente a los Problemas Nacionales. Colección Pensamiento Nacional. Argentina: Ediciones UNLa.

Jiménez, E.

2008. La revista científica y el quehacer académico. Anatomía y fisiología de las revistas científicas arbitraje y costo de las revistas científicas. Bitácora-e: Revista Electrónica Latinoamericana de Estudios Sociales, Históricos y Culturales de la Ciencia y la Tecnología, 2008(1): 146-152.

Jump, P.

2013. Personnel 'Can't be Chosen on Citations Alone'. Disponible en : http://www.timeshighereducation.co.uk/ news/personnel-cant-be-chosen-on-citations-alone/2001208. article Consultado: 15 /Agosto/ 2014.

McVeigh, $M$.

2002. Journal self-citation in the Journal Citation Reports Science Edition (2002). Disponible: http://wokinfo.com/ essays/journal-self-citation-jcr/Consultado: 15/agosto/2014.

Molina-Montenegro, M.; Gianoli, E.

2010. El índice-I, un nuevo estimador del impacto de la productividad científica: Los ecólogos de Chile como caso de estudio. Revista Chilena de Historia Natural, 83: 219-227. 
Origgi, G.; Ramello, G. B.

2015. Current Dynamics of Scholarly Publishing. Evaluation review, 39(1): 3-18.

Riaza, F.

2002. La transmisión del conocimiento. Revista de Estudios de Ciencias Sociales y Humanidades de Córdova, 8: 11-14. Schweizer, K.

2015. Judging a journal by the impact factor: Is it appropriate and fair for assessment journals? European Journal of Psychological Assessment, 26(4): 235-237.

Székely, T.; Krüger, O.; Krause, E.

2014. Errors in Science: The Role of Reviewers. Trends in Ecology \& Evolution, 29(7): 371-373.

Villatoro, F.

2011. El Vicio de las Autocitas. Disponible en: http://francis. naukas.com/2011/06/16/el-vicio-de-las-autocitas/ Consultado: 23/agosto / 2014
Villatoro, F.

2012. El Bueno, el Malo y el Feo en las Revistas de Acceso Gratuito (open access). Disponible en: http://francis.naukas. com/2012/11/26/el-bueno-el-malo-y-el-feo-en-las-revistasde-acceso-gratuito-open-access/Consultado: 23/agosto/2014. Villatoro, F.

2013a. Descubierta una Red de Autocitas entre Cuatro Revistas Brasileñas. Disponible en: http://francis.naukas. com/2013/08/28/descubierta-una-red-de-autocitas-entrecuatro-revistas-brasilenas/ Consultado: 23/ agosto/2014.

Villatoro, F.

2013b. La falta de rigor de Thomson Reuters al calcular el indice de impacto de una revista en el JCR. Disponible en: http://www.observatorioderevistascientificas.com/index. $\mathrm{php} /$ noticias/180-la-falta-de-rigor-de-thomson-reutersal-calcular-el-indice-de-impacto-de-una-revista-en-el-jcr Consultado: 23/agosto/2014. 
